\title{
Quality Time Tradeoff Operator for Designing Efficient Multi Level Genetic Algorithms
}

\author{
George G. Mitchell \\ RINCE \\ Dublin City University \\ Ireland \\ george.mitchell \\ @dcu.ie
}

\author{
Barry McMullin \\ RINCE \\ Dublin City University \\ Ireland \\ barry.mcmullin \\ @dcu.ie
}

\author{
James Decraene \\ RINCE \\ Dublin City University \\ Ireland \\ james.decraene \\ @eeng.dcu.ie
}

\author{
Ciaran Kelly \\ RINCE \\ Dublin City University \\ Ireland \\ ciaran.kelly \\ @computing.dcu.ie
}

\begin{abstract}
We present a novel cost benefit operator that assists multi level genetic algorithm searches. Through the use of the cost benefit operator, it is possible to dynamically constrain the search of the base level genetic algorithms, to suit the user's requirements. We note that the current literature has abundant studies on meta evolutionary GA's, however these approaches have not identified an efficient approach to the termination of base GA searchs or a means to balance practical consideration such as quality of solution and the expense of computation. Our Quality time tradeoff operator (QTT) is user defined, and acts as a base level termination operator and also provides a fitness value for the meta-level GA. In this manner, the amount of computation time spent on less encouraging configurations can be specified by the user. Our approach was applied to a computationally intensive test problem which evaluates a large set of configuration settings for the base GAs to find suitable configuration settings (population size, crossover operator and rate, mutation operator and rate, repair or penalty and the use of adaptive mutation rates) for selected TSP problems.
\end{abstract}

\section{Categories and Subject Descriptors}

I.2.8 [Artificial Intelligence]: Problem Solving, Control

Methods, and Search-Heuristic methods

\section{General Terms}

algorithms, performance, design

\section{Keywords}

Cost benefit operator, Multi level Genetic Algorithms, fitness and termination.

\section{QTT}

Sosič [1] developed a tradeoff operator for a local optimization algorithm, Duty (as termed by Sosič) which minimized the excess (error) of a present solution compared to the benchmark known optimal solution. Our QTT operator utilized in the experiment of the multi level GA was as follows:

$$
\operatorname{tradeoff}(\mathrm{Q})=\mathrm{T}+W(\mathrm{Q})
$$

Where $\mathrm{T}$, the computation time is measured as the number of generations, $\mathrm{Q}$ the quality of the solution is the raw fitness value for the individual solution and $\mathrm{W}$ the weighting factor placed on the quality of the solution. By weighting the quality of solutions

Copyright is held by the author/owner(s). GECCO'07, July 7-11, 2007, London, England, United Kingdom ACM 978-1-59593-697-4/07/0007. the tradeoff operator can be set by the user to terminate the solution at the desired cost benefit point (where time spent searching for a better solution does not out weight the improvement in the quality of the solution). The tradeoff operator should produce a ' $u$ ' shaped curve, by identifying the minimum of this curve it is possible to halt the base level GA search. This is achieved through two key mechanisms:

1. A low pass filter (for a minimization problem) is applied to the solution fitness values generated by the base level GA. This determines the best solution found so far. This best solution is computed in real-time.

2. A sliding window grace period is applied. This gives an opportunity to encounter fitter candidate solutions. The size of the window is user defined and affects the running time of the search. Typically a small window returns a result more rapidly. This is tightly coupled to the quality time tradeoff.

\section{RESULTS}

Results indicate that in this instance when the QTT is applied to the selecting of configurations for GAs to solve the TSP a set of operators and associated parameters for individual TSP problems exist rather than one single configuration setting suiting all of the tested problems (50, 70 and 100 city respectively). The weighting for the tradeoff operator was varied widely so as to consider a wide range of values. It is important to note that these tests were not performed to produce optimal results but rather to find configurations that meet the user's quality time tradeoff requirements as specified through the QTT settings.

Constraints for the problem could be satisfied by validity preserving crossover and mutation operators or when non validity preserving operators were employed one of 25 possible repair [2] techniques could be employed, a weighted penalty function to penalize invalid solutions was also considered. Results indicate that the multi level GA found that a combination of repair methods was consistently the most effective.

\section{REFERENCES}

[1] R. Sosic and G. D. Wilby, "Using the Quality-Time Tradeoff in Local optimization.," Proceedings of the IEEE Second ANZIIS Conference, pp. 253- 257, 1994.

[2] G. G. Mitchell, "Validity Constraints and the TSP GeneRepair of Genetic Algorithms.," Artificial Intelligence and Applications, pp. 306-311, 2005. 